\title{
Inflammatory Markers of the Systemic Capillary Leak Syndrome (Clarkson Disease)
}

\section{Citation}

Xie, Zhihui, Eunice Chan, Yuzhi Yin, Chandra C. Ghosh, Laura Wisch, Celeste Nelson, Michael Young, Samir M. Parikh, and Kirk M. Druey. 2014. "Inflammatory Markers of the Systemic Capillary Leak Syndrome (Clarkson Disease)." Journal of clinical \& cellular immunology 5 (1): 1000213. doi:10.4172/2155-9899.1000213. http://dx.doi.org/10.4172/2155-9899.1000213.

\section{Published Version}

doi:10.4172/2155-9899.1000213

\section{Permanent link}

http://nrs.harvard.edu/urn-3:HUL.InstRepos:13454768

\section{Terms of Use}

This article was downloaded from Harvard University's DASH repository, and is made available under the terms and conditions applicable to Other Posted Material, as set forth at http:// nrs.harvard.edu/urn-3:HUL.InstRepos:dash.current.terms-of-use\#LAA

\section{Share Your Story}

The Harvard community has made this article openly available.

Please share how this access benefits you. Submit a story.

\section{Accessibility}




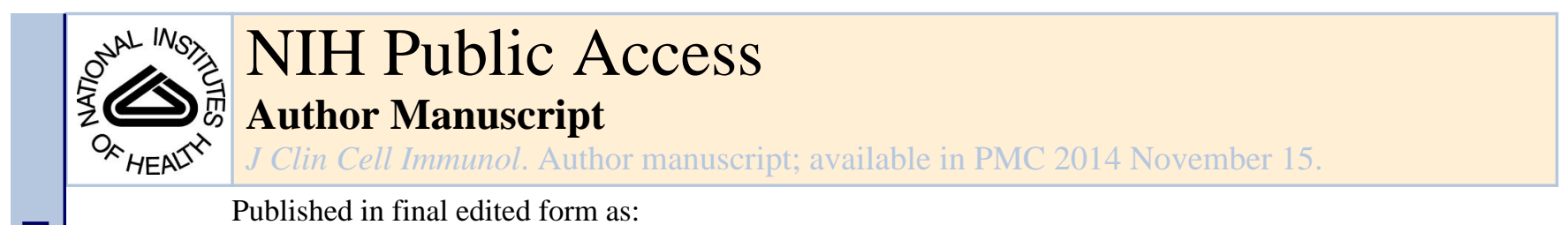

Published in final edited form as:

J Clin Cell Immunol. 2014 ; 5: 1000213-. doi:10.4172/2155-9899.1000213.

\title{
Inflammatory Markers of the Systemic Capillary Leak Syndrome (Clarkson Disease)
}

\author{
Zhihui Xie ${ }^{1,{ }^{*}}$, Eunice Chan ${ }^{1}$, Yuzhi Yin ${ }^{1}$, Chandra C. Ghosh ${ }^{2}$, Laura Wisch ${ }^{1}$, Celeste \\ Nelson ${ }^{1}$, Michael Young ${ }^{3}$, Samir M. Parikh ${ }^{2}$, and Kirk M. Druey ${ }^{1}$ \\ ${ }^{1}$ Laboratory of Allergic Diseases, NIAID/NIH, Bethesda, MD, USA \\ ${ }^{2}$ Department of Medicine, Division of Nephrology and Center for Vascular Biology Research, Beth \\ Israel Deaconess Medical Center and Harvard Medical School, Boston, MA, USA \\ ${ }^{3}$ Clinical Research Directorate/CMRP, Leidos Biomedical Research Inc., Frederick, National \\ Laboratory for Cancer Research, Frederick, MD, USA
}

\begin{abstract}
Objectives-The Systemic Capillary Leak Syndrome (SCLS) is a rare and potentially fatal disorder resembling systemic anaphylaxis that is characterized by transient episodes of hypotensive shock and peripheral edema. The pathogenesis of SCLS is unknown, and triggers for attacks are apparent only in a minority of patients. We introduce a clinical algorithm for the diagnosis of SCLS, and we investigated potential serum biomarkers of acute SCLS episodes.

Methods-We analyzed serum cytokines in a cohort of 35 patients with an established diagnosis of SCLS and characterized the effects of SCLS sera on endothelial cell function. We investigated the cellular source(s) of CXCL10, a chemokine that was significantly elevated in both basal and acute SCLS sera, by flow cytometry.
\end{abstract}

Results-Several cytokines were elevated in acute SCLS sera compared to baseline or sera from healthy controls, including CXCL10, CCL2, IL-1 $\beta$, IL-6, IL-8, IL-12 and TNFa. The majority of acute sera failed to activate endothelial cells as assessed by surface adhesion marker expression. Monocytes appear to be the major source of serum CXCL10, and the percentage of CXLC10+ monocytes in response to IFN $\gamma$ stimulation was increased in SCLS subjects compared to controls.

Conclusions-The presence of proinflammatory cytokines in acute SCLS sera suggests that inflammation or infection may have a role in triggering episodes. The enhanced capacity of monocytes from SCLS patients to produce CXCL10 suggests a new therapeutic avenue for SCLS.

\section{Keywords}

Systemic capillary leak syndrome; Inflammation; Cytokines; CXCL10; Monocytes

Copyright: @ 2014 Xie Z, et al.

This is an open-access article distributed under the terms of the Creative Commons Attribution License, which permits unrestricted use, distribution, and reproduction in any medium, provided the original author and source are credited.

"Corresponding author: Zhihui Xie, 10 Center Drive, Room 11N242 Bethesda, MD 20892, USA, Tel: 301-594-7522; Fax:

301-480-8384; xiez@niaid.nih.gov.

Financial disclosures

The authors declare no competing financial interests. 


\section{Introduction}

The Systemic Capillary Leak Syndrome (SCLS) is a life-threatening disorder that occurs sporadically, typically in middle-aged Caucasians $[1,2]$. SCLS is characterized by recurrent, reversible episodes of distributive shock and anasarca due to sudden and unexplained leakage of plasma into the tissues. SCLS resembles, and is often erroneously diagnosed as, more common disorders such as sepsis, angioedema, and systemic anaphylaxis. Although a monoclonal gammopathy of unknown significance (MGUS) is highly associated with SCLS $[3,4]$, its pathophysiologic significance is unclear. Therefore, because no specific biomarkers or pathognomonic clinical sign(s) of SCLS exist currently, the triad of transient hypotension, hemoconcentration, and serum hypoalbuminemia in the absence of underlying causes is sufficient to establish the diagnosis [2,5,6]. Fewer than 250 cases have been reported since Clarkson described SCLS in 1960 [2]. There is no specific treatment for acute SCLS other than hemodynamic support with intravenous fluids and vasopressors. Drugs such as theophylline, $\beta 2$-adrenergic agonists, and most recently, imatinib, have been used as prophylactic agents to promote vascular integrity in SCLS [6]. Based on anecdotal evidence accumulated over several years, it has become apparent that monthly intravenous immunoglobulin (IVIG, $2 \mathrm{~g} / \mathrm{kg}$ ) infusions have severely curtailed attacks in a majority of patients who receive this therapy indefinitely [6-8].

Most research findings on SCLS have been based on single case reports, or purely clinical observations [2,6]. Using samples from our (then) 23-patient cohort, we reported previously that sera from SCLS patients during acute disease intervals uniformly induced hyperpermeability of human microvascular endothelial cell (HMVEC) monolayers due to disruption of endothelial adherens junctions and actin stress fiber formation [4]. In contrast, sera from these patients obtained during remission had little to no impact on HMVEC barrier integrity and resembled sera from healthy controls. HMVEC hyperpermeability elicited by acute SCLS sera was inhibited by pretreatment of target endothelial cells with IVIG or by antibody neutralization of Angiopoietin-2 (Ang2), a known mediator of vascular permeability that was elevated in acute SCLS sera compared to sera from healthy donors [4].

Although the trigger(s) of acute SCLS episodes are unclear, a longitudinal case registry study of 28 SCLS patients found that about three quarters of subjects experienced flu-like symptoms prior to attack onset [6]. Analysis of our expanded cohort (35 subjects) revealed elevated levels of several proinflammatory cytokines in acute sera, particularly CXCL10. Monocytes from a subset of these patients demonstrated an increased capacity for CXCL10 production. These results suggest a potential role for CXCL10 in the initiation of acute SCLS.

\section{Materials and Methods}

\section{Study subjects}

Patients were diagnosed with SCLS and classified according to established criteria described previously [2,6]. Patients were seen at the Clinical Center of the National Institutes of Health. Written informed consent was obtained from each patient, and the study protocol 
(09-I-0184) conformed to the ethical guidelines of the 2008 Declaration of Helsinki as reflected in a priori approval from the Institutional Review Board of the National Institute of Allergy and Infectious Diseases of NIH. Age-, sex-, and race-matched serum samples and lymphapheresis samples were obtained from the NIH Blood Bank.

\section{Cells and reagents}

Human umbilical vascular endothelial cells (HUVECs) were purchased from American Tissue Type Collection. Endothelial Growth Medium (EGM) 2 was obtained from Lonza. AlexaFluor 647-conjugated anti-ICAM1, phycoerythryin-conjugated anti-VCAM1, antiCXCL10, phycoerythryin/Cy7-conjugated anti-CD14 antibodies and the protein transport inhibitor monensin were purchased from BioLegend. Phycoerythryin-Cy5-conjugated antiE-selectin and APC-conjugated anti-CD19 antibodies were obtained from BD Biosciences. Qdot 605-conjugated anti-CD3 and Live/Dead fixable violet dead stain kit were from Life Technologies. Recombinant human TNFa and IFN $\gamma$, and CXCL10 Quantikine ELISA kit were purchased from R \& D Systems.

\section{Cytokine measurements}

Serum cytokines were measured using a Bio-plex Pro human cytokine 27-plex and a customized human cytokine 9-plex assay kit purchased from Bio-Rad according to the manufacturer's instructions.

\section{Endothelial activation marker analysis}

HUVECs were seeded in 6-well plate and incubated overnight at $37^{\circ} \mathrm{C}$. Cells were incubated with test serum $(10 \% \mathrm{vol} / \mathrm{vol}$ final concentration) in growth medium for 4 hours. The cells were then detached with CellStripper (Mediatech), washed once with PBS, and stained with the indicated antibodies for 30 minutes. Cells were then washed twice, fixed with $4 \%$ paraformaldehyde for 10 minutes, and analyzed by flow cytometry.

\section{CXCL10 measurement by ELISA}

HUVECs were seeded in 96-well plate overnight at $37^{\circ} \mathrm{C}$, and followed by incubation with test sera ( $10 \% \mathrm{vol} / \mathrm{vol}$ final concentration) in growth medium for 24 hours. Cell culture supernatants were collected and analyzed for CXCL10 by ELISA.

\section{PBMC isolation}

Peripheral blood mononuclear cells (PBMCs) were isolated from lymphapheresis samples using lymphocyte separation medium (LSM, LP BioMedicals). Briefly, apheresis samples were diluted 1:1 in PBS, and $30 \mathrm{ml}$ of diluted sample was overlaid on onto LSM (15 ml) in a $50 \mathrm{ml}$ conical tube. Samples were centrifuged at $800 \times \mathrm{g}$ in a tabletop centrifuge at room temperature for 40 minutes with break off.

\section{Flow cytometric analysis of CXCL10 production}

PBMCs were left untreated or stimulated with IFN $\gamma(20 \mathrm{ng} / \mathrm{ml})$ in the presence of monensin overnight in $37^{\circ} \mathrm{C}$. The following day cells were stained with Live/Dead violet staining kit according to manufacturer's instructions and fixed in $4 \%$ paraformaldehyde. Fixed cells 
were permeabilized with blocking buffer $\left(0.1 \%\right.$ saponin, $1 \mathrm{mM} \mathrm{CaCl}_{2}, 1 \mathrm{mM} \mathrm{MgSO}_{4}, 0.1 \%$ BSA, 10 mM HEPES, 5\% milk, and 5\% human Fc receptor inhibitor, in PBS) for 30 minutes followed by staining with antibodies in blocking buffer for additional $30 \mathrm{~min}$. Stained cells were washed twice and analyzed using LSRII flow cytometer (BD BioSciences).

\section{Statistical analysis}

Data were analyzed with the GraphPad Prism 6 software package. 1-way ANOVA or Kruskal-Wallis (for multiple groups) was used for grouped cytokine analyses, and unpaired parametric Student's $t$ test was used for flow analysis of CXCL10 production. $P$ values < 0.05 were considered significant.

\section{Results}

\section{Algorithm for the diagnosis of SCLS}

SCLS should be considered in a patient with unexplained, transient hypotension and/or peripheral edema (Figure 1). If a temporally linked exposure (e.g. food, insect venom, drug) is suspected, elevated serum tryptase should exclude systemic anaphylaxis. Although our experience and that of others [6,9] suggest that acute triggers for SCLS attacks are often absent, viral-type upper respiratory and/or systemic symptoms may be present in many patients with SCLS prior to the onset of an episode. While catastrophic SCLS attacks are typically accompanied by massive edema of the face, trunk, and peripheral extremities, swelling resembling angioedema may be confined to certain areas (periorbital, back, and abdomen) in less severe episodes. Thus, complement factor 1 esterase inhibitor (C1 INH) levels and function should be evaluated in all patients with suspected SCLS to rule out hereditary or acquired angioedema.

A hallmark of severe acute SCLS episodes is hemoconcentration due to the loss of water and solutes into the extravascular space. Marked elevations in serum hemoglobin over the patient's baseline, often greater than $20 \mathrm{~g} / \mathrm{dL}$, are common, occasionally leading to an erroneous diagnosis of polycythemia vera [10]. In contrast to cases of dehydration and sepsis, the hemoconcentration and hypotension of SCLS do not reverse immediately following administration of intravenous fluids and/or vasopressors. Central venous pressures remain low (typically $<2 \mathrm{~mm} \mathrm{Hg}$ ) in the acute SCLS leak phase, and massive intravenous saline infusion often aggravates peripheral edema and can elicit compartment syndromes in the extremities.

Serum hypoproteinemia due to protein extravasation is universally present in acute SCLS, and albumin levels of $<2 \mathrm{~g} / \mathrm{dL}$ are common. Some patients may present with persistent, noncyclical, peripheral edema and hypoalbuminemia, and this subset may or may not experience acute hypotensive episodes. These patients are classified as having "chronic" SCLS and may also present with visceral (pleural, pericardial) effusions. Finally, it should be emphasized that although MGUS is present in $85-95 \%$ of SCLS cases, it is not required for the diagnosis. 


\section{Characteristics of the SCLS study cohort}

We evaluated 35 patients with a confirmed diagnosis of SCLS based on the criteria outlined above and exclusion of other primary causes of hypotension and/or edema (Table 1). The median age at the time of the SCLS diagnosis was 46 years (range 22 months to 66 years) although the vast majority of patients experienced clinical symptoms attributable to SCLS for several years prior to receiving the actual diagnosis. All but one patient was Caucasian, and $43 \%$ were female. Our cohort included three children and three adult patients with chronic edema. Most subjects experienced at least two episodes of SCLS per year, but the range varied widely, from once in five years to weekly. Overall, 86\% had MGUS, and 27/29 M-proteins were of the IgG isotype. M-proteins were not detected in any of the children, whereas they were present in $91 \%$ of adults with SCLS. While most patients were treated with theophylline and terbutaline following diagnosis, 79\% (23/29) experienced breakthrough episodes while on this regimen. Currently, $63 \%$ are treated with monthly infusions of IVIG (2 $\mathrm{g} / \mathrm{kg})$, and the majority of these patients (20/22) have been essentially episode-free for periods of up to eight years. One patient, a seven-year old child, is receiving subcutaneous IG because of IVIG intolerance. Two somewhat atypical patients (Pt. 3 and Pt. 31) experienced recurrent episodes of idiopathic hypotension, hemoconcentration, and hypoalbuminemia consistent with the diagnosis of SCLS, yet presented with unique symptomatology and did not respond at all to standard SCLS therapy, including IVIG. These patients also had M-protein isotypes unusual for SCLS (IgA or IgM, respectively).

\section{Serum cytokine analysis of SCLS}

Although nearly one quarter of subjects reported flu-like symptoms prior to the onset of a full-blown attack [4], whether this prodrome represents a concomitant infection or is a primary feature of SCLS is unknown. Our previous studies that include a cohort of 23 SCLS subjects demonstrated that some (VEGF, Ang 2), but not all (IL-2), soluble mediators of inflammation were increased in acute SCLS sera [4]. To assess the role of infection and/or inflammation in acute SCLS episodes and to discover new mediators that may contribute to disease onset and/or severity, we analyzed a panel of 27 serum cytokines in sera from 31 SCLS patients in remission, 14 SCLS patients with acute symptoms (samples were not available from all patients), and 37 healthy controls by multiplex bead assay. Ten cytokines (IL-2, IL-4, IL-5, IL-10, IL-13, IL-15, IL-17, G-CSF, GM-CSF, CCL3) were absent or present at very low levels in most sera tested, while levels of the cytokine RANTES were present at levels above the detectable assay range in all samples. Quantities of eight cytokines (IL-1RA, IL-7, IL-9, bFGF, PDGFb, CCL4, and CCL11) did not differ significantly among the groups or between individual SCLS subjects. Consistent with our previous findings, VEGF was increased in acute SCLS sera compared with sera from healthy controls (Figure 2A). We detected significantly increased levels of seven cytokines in acute SCLS sera relative to sera from healthy donors, specifically, CXCL10, CCL2, IL-12, IL-1 $\beta$, IL-6, IL-8, and TNFa (Figures 2B-2H). Of all cytokines measured, only CXCL10 was significantly increased both in baseline and acute SCLS sera relative to controls (Figure 2B). These data suggested that TH1-associated inflammation or infection is associated with acute SCLS episodes. 


\section{Activation of vascular endothelial cells by acute SCLS sera}

Skin biopsies of SCLS patients have revealed the presence of perivascular leukocytic inflammation during acute episodes in some cases [11]. Proinflammatory cytokines including TNFa and IL-8 have been shown to increase adhesion molecule surface expression on endothelial cells, which in turn promotes leukocyte rolling, migration and extravasation [12]. To determine whether factors in SCLS sera could evoke endothelial barrier dysregulation by inducing leukocyte adhesion, we incubated HUVECs with acute and baseline sera from SCLS subjects or healthy donors and analyzed surface expression of E-selectin, VCAM-1 and ICAM-1 by flow cytometry. Unexpectedly, among the SCLS sera tested ( 20 baseline and 13 acute) only Pt. 3's episodic serum induced upregulation of these adhesion molecules in HUVECs (Figure 3A-B). Surface expression of these markers induced by Pt. 3's episodic sera was comparable to that evoked by TNFa (Figure 3A, dotted line) [13], a well-known endothelial activator that served as positive control for these experiments. The concentration of TNFa in Pt. 3' episodic serum (top square dot in Figure $2 \mathrm{G}$ ) is comparable to that used in control experiments, 20 -fold higher than that found in most of other SCLS sera (Figure 2G). These results and the unresponsiveness of HUVECs to acute SCLS serum samples containing low levels of TNFa suggest that TNFa is probably the cytokine that mediated endothelial adhesion/activation marker upregulation in Pt. 3's episodic sera. Since endothelial cells have the capacity to produce CXCL10 in response to inflammatory stimuli [14], we examined whether they produced CXCL10 following treatment with SCLS sera. We incubated HUVECs with acute or baseline sera from healthy controls or subjects with SCLS for 24 hours and measured CXCL10 in culture supernatants. TNFa plus IFN $\gamma$ served as a positive control, as described in previous work [14,15]. None of the SCLS sera tested elicited CXCL10 secretion from HUVECs (Figure 3C). Taking together, these results suggested that in classic SCLS, endothelium is probably not the major source of CXCL10.

\section{Increased CXCL10-producing monocytes in SCLS subjects}

CXCL10, which is induced by many proinflammatory factors such as IFN $\gamma$, is secreted by many cell types including monocytes, endothelial cells, and fibroblasts during inflammation or infections [16]. To determine the cellular source of CXCL10 in SCLS sera, we studied whether CXCL10 production by peripheral blood mononuclear cells differed in control subjects and those with SCLS. As expected, percentages of PBMCs producing CXCL10 constitutively were low (data not shown), but INF $\gamma$ elicited dramatic increases in the number of CXCL10 $0^{+}$cells (Figure 4A). Whereas percentages of CXCL10 ${ }^{+} \mathrm{T}$ and B lymphocytes were low, about $30 \%$ of monocytes from healthy control subjects produced CXCL10 following IFN $\gamma$ stimulation. Greater than $90 \%$ of all CXCL10-producing non-B non-T cells were monocytes $\left(\mathrm{CD} 14^{+}\right)$(Figure 4A, lower right panel). Following IFN $\gamma$ treatment, we detected a significantly higher number of CXCL10 ${ }^{+}$mononuclear cells and CXCL10 ${ }^{+}$ monocytes in SCLS subjects compared to healthy controls (Figures 4B-4C). These results suggested that monocytes from subjects with SCLS have increased responsiveness to an inflammatory stimulus (IFN $\gamma$ ). 


\section{Discussion}

Among an extensive group of proinflammatory cytokines studied, we found that elevations

in CCL2, IL-1 $\beta$, IL-6, IL8, IL-12 and TNFa were associated with acute SCLS episodes, and CXCL10 levels were both constitutively and acutely increased in SCLS sera. CXCL10 appears to be derived principally from peripheral monocytes, and SCLS patients have significantly more circulating monocytes with the capacity to make CXCL10 than controls.

We demonstrated increased VEGF levels in acute SCLS sera, which corroborated our previous ELISA results in sera from nine SCLS subjects (also included in the current group) [4], indicating that VEGF is a potential mediator of endothelial barrier dysfunction in SCLS. Although symptoms associated with viral infections do not precede SCLS episodes in all cases, some patients report episodes triggered by infections. Accordingly, we found increased quantities of CCL2, IL-1 $\beta$, IL-6, IL-8, IL-12 and CXCL10 in SCLS acute sera, suggesting that type 1 immune responses $\mathrm{T}_{\mathrm{H}} 1$ (e.g. chronic viral, bacterial, or protozoan infections), rather than $\mathrm{T}_{\mathrm{H}} 2$ allergic immunity, may contribute the SCLS phenotype in at least a fraction of patients.

Of all cytokines studied, CXCL10 was prominently elevated in both basal and acute SCLS sera relative to controls. CXCL10 has been shown to inhibit endothelial cell proliferation and induce apoptosis [17], a mechanism previously implicated in the pathogenesis of SCLS $[18,19]$. However, we were unable to demonstrate endothelial apoptosis induced by acute SCLS sera in our previous study [4]. In the current work, acute SCLS sera failed to activate endothelial cell adhesion marker expression (with one exception), and none of the sera elicited CXCL10 production by endothelial cells, suggesting that endothelial cells may not be a major source of CXCL10 in SCLS. Although IFN $\gamma$ is a strong inducer of CXCL10, many $\mathrm{T}_{\mathrm{H}} 1$-associated cytokines are able to increase CXCL10 production by monocytes and macrophages. Because IFN $\gamma$ levels were similar in SCLS and control sera, other factors including VEGF, TNFa, IL- $\beta$ and IFN $\gamma$ may act synergistically to elicit CXCL10 production by monocytes/macrophages in SCLS [20-22]. IFN $\gamma$-independent CXCL10 production may also occur [23]. For example, a single polynucleotide polymorphism (SNP) in the Cxcllo promoter $(-135 \mathrm{G}>\mathrm{A})$ enhanced its transcriptional activity in tuberculosis [24]. Thus, mutations or polymorphisms in Cxcl10 could also account for aberrant CXCL10 production in SCLS.

Because elevated serum levels of CXCL10 have been observed in many diseases including rheumatoid arthritis, systemic lupus erythematosus, systemic sclerosis [25], and HIV infection [16], it probably has no utility as a specific diagnostic marker for SCLS. However, it may be useful for diagnosis and/or prognosis when considered with previously identified permeability mediators VEGF and Ang2. CXCL10 is a potent chemoattractant for monocytes/macrophages and activated $\mathrm{T}$ cells and promotes leukocyte transmigration through endothelium into inflamed tissues. Further study of abnormal CXCL10 production and its cellular source(s) may provide insights into the initial permeability-inducing trigger in SCLS episodes as well as uncover new therapeutic strategies for this potentially fatal disorder. 


\section{Acknowledgments}

We thank Dr. Helene Rosenberg for helpful discussions and critical review of the manuscript.

Funding

This work was supported by the Intramural Research Program of the National Institute of Allergy and Infectious Diseases, National Institutes of Health (Project AI001830, K.M.D). Support by M.Y. for this project was funded in whole or in part with federal funds from the National Cancer Institute, National Institutes of Health, under Contract No. HHSN261200800001E. S.M.P. is supported by grants from the National Institutes of Health (R01-HL093234 and R01-DK095072) and the American Diabetes Association (1-13-BS-1-41).

\section{Abbreviations}

$\begin{array}{ll}\text { SCLS } & \text { Systemic Capillary Leak Syndrome } \\ \text { HUVEC } & \text { Human Umbilical Vein Endothelial Cells } \\ \text { PBMC } & \text { Peripheral Blood Mononuclear Cells } \\ \text { ICAM-1 } & \text { Intercellular Adhesion Molecule-1 } \\ \text { VCAM-1 } & \text { Vascular Cell Adhesion Molecule-1 } \\ \text { IVIG } & \text { Intravenous Immunoglobulin } \\ \text { IFN } \gamma & \text { Interferon Gamma } \\ \text { TNFa } & \text { Tumor Necrosis Factor a } \\ \text { VEGF } & \text { Vascular Endothelial Growth Factors } \\ \text { Ang2 } & \text { Angiopoietin 2 } \\ \text { MGUS } & \text { Monoclonal Gammopathy of Unknown Significance }\end{array}$

\section{References}

1. CLARKSON B, THOMPSON D, HORWITH M, LUCKEY EH. Cyclical edema and shock due to increased capillary permeability. Am J Med. 1960; 29:193-216. [PubMed: 13693909]

2. Druey KM, Greipp PR. Narrative review: the systemic capillary leak syndrome. Ann Intern Med. 2010; 153:90-98. [PubMed: 20643990]

3. Kapoor P, Greipp PT, Schaefer EW, Mandrekar SJ, Kamal AH, et al. Idiopathic systemic capillary leak syndrome (Clarkson's disease): the Mayo clinic experience. Mayo Clin Proc. 2010; 85:905912. [PubMed: 20634497]

4. Xie Z, Ghosh CC, Patel R, Iwaki S, Gaskins D, et al. Vascular endothelial hyperpermeability induces the clinical symptoms of Clarkson disease (the systemic capillary leak syndrome). Blood. 2012; 119:4321-4332. [PubMed: 22411873]

5. Dhir V, Arya V, Malav IC, Suryanarayanan BS, Gupta R, et al. Idiopathic systemic capillary leak syndrome (SCLS): case report and systematic review of cases reported in the last 16 years. Intern Med. 2007; 46:899-904. [PubMed: 17575386]

6. Gousseff M, Arnaud L, Lambert M, Hot A, Hamidou M, et al. The systemic capillary leak syndrome: a case series of 28 patients from a European registry. Ann Intern Med. 2011; 154:464471. [PubMed: 21464348]

7. Abgueguen P, Chennebault JM, Pichard E. Immunoglobulins for treatment of systemic capillary leak syndrome. Am J Med. 2010; 123:e3-4. [PubMed: 20569743]

8. Pecker M, Adams M, Graham W. The systemic capillary leak syndrome. Ann Intern Med. 2011; 155:335. [PubMed: 21893630] 
9. Tahirkheli NK, Greipp PR. Treatment of the systemic capillary leak syndrome with terbutaline and theophylline. A case series. Ann Intern Med. 1999; 130:905-909. [PubMed: 10375339]

10. Doubek M, Brychtova Y, Tomiska M, Mayer J. Idiopathic systemic capillary leak syndrome misdiagnosed and treated as polycythemia vera. Acta Haematol. 2005; 113:150-151. [PubMed: 15802896]

11. Cicardi M, Berti E, Caputo V, Radice F, Gardinali M, et al. Idiopathic capillary leak syndrome: evidence of CD8-positive lymphocytes surrounding damaged endothelial cells. J Allergy Clin Immunol. 1997; 99:417-419. [PubMed: 9058699]

12. Aranda JF, Reglero-Real N, Marcos-Ramiro B, Ruiz-Sáenz A, Fernández-Martín L, et al. MYADM controls endothelial barrier function through ERM-dependent regulation of ICAM-1 expression. Mol Biol Cell. 2013; 24:483-494. [PubMed: 23264465]

13. Madge LA, Pober JS. TNF signaling in vascular endothelial cells. Exp Mol Pathol. 2001; 70:317325. [PubMed: 11418010]

14. Indraccolo S, Pfeffer U, Minuzzo S, Esposito G, Roni V, et al. Identification of genes selectively regulated by IFNs in endothelial cells. J Immunol. 2007; 178:1122-1135. [PubMed: 17202376]

15. Proost P, Struyf S, Loos T, Gouwy M, Schutyser E, et al. Coexpression and interaction of CXCL10 and CD26 in mesenchymal cells by synergising inflammatory cytokines: CXCL8 and CXCL10 are discriminative markers for autoimmune arthropathies. Arthritis Res Ther. 2006; 8:R107. [PubMed: $16846531]$

16. Liu M, Guo S, Hibbert JM, Jain V, Singh N, et al. CXCL10/IP-10 in infectious diseases pathogenesis and potential therapeutic implications. Cytokine Growth Factor Rev. 2011; 22:121130. [PubMed: 21802343]

17. Green LA, Petrusca D, Rajashekhar G, Gianaris T, Schweitzer KS, et al. Cigarette smoke-induced CXCR3 receptor up-regulation mediates endothelial apoptosis. Am J Respir Cell Mol Biol. 2012; 47:807-814. [PubMed: 22936405]

18. Assaly R, Olson D, Hammersley J, Fan PS, Liu J, et al. Initial evidence of endothelial cell apoptosis as a mechanism of systemic capillary leak syndrome. Chest. 2001; 120:1301-1308. [PubMed: 11591575]

19. Johansson BR, Löfdahl CG. Ultrastructure of the microvessels in skeletal muscle in a case of systemic capillary leak syndrome. Acta Med Scand. 1979; 206:413-416. [PubMed: 525443]

20. Boulday G, Haskova Z, Reinders ME, Pal S, Briscoe DM. Vascular endothelial growth factorinduced signaling pathways in endothelial cells that mediate overexpression of the chemokine IFN-gamma-inducible protein of $10 \mathrm{kDa}$ in vitro and in vivo. J Immunol. 2006; 176:3098-3107. [PubMed: 16493069]

21. Yeruva S, Ramadori G, Raddatz D. NF-kappaB-dependent synergistic regulation of CXCL10 gene expression by IL-1beta and IFN-gamma in human intestinal epithelial cell lines. Int J Colorectal Dis. 2008; 23:305-317. [PubMed: 18046562]

22. Lombardi A, Cantini G, Mello T, Francalanci M, Gelmini S, et al. Molecular mechanisms underlying the pro-inflammatory synergistic effect of tumor necrosis factor alpha and interferon gamma in human microvascular endothelium. Eur J Cell Biol. 2009; 88:731-742. [PubMed: 19782427]

23. Brownell J, Bruckner J, Wagoner J, Thomas E, Loo YM, et al. Direct, interferon-independent activation of the CXCL10 promoter by NF-kappaB and interferon regulatory factor 3 during hepatitis C virus infection. J Virol. 2014; 88:1582-1590. [PubMed: 24257594]

24. Tang NL, Fan HP, Chang KC, Ching JK, Kong KP, et al. Genetic association between a chemokine gene CXCL-10 (IP-10, interferon gamma inducible protein 10) and susceptibility to tuberculosis. Clin Chim Acta. 2009; 406:98-102. [PubMed: 19523460]

25. Lee EY, Lee ZH, Song YW. CXCL10 and autoimmune diseases. Autoimmun Rev. 2009; 8:379_ 383. [PubMed: 19105984] 


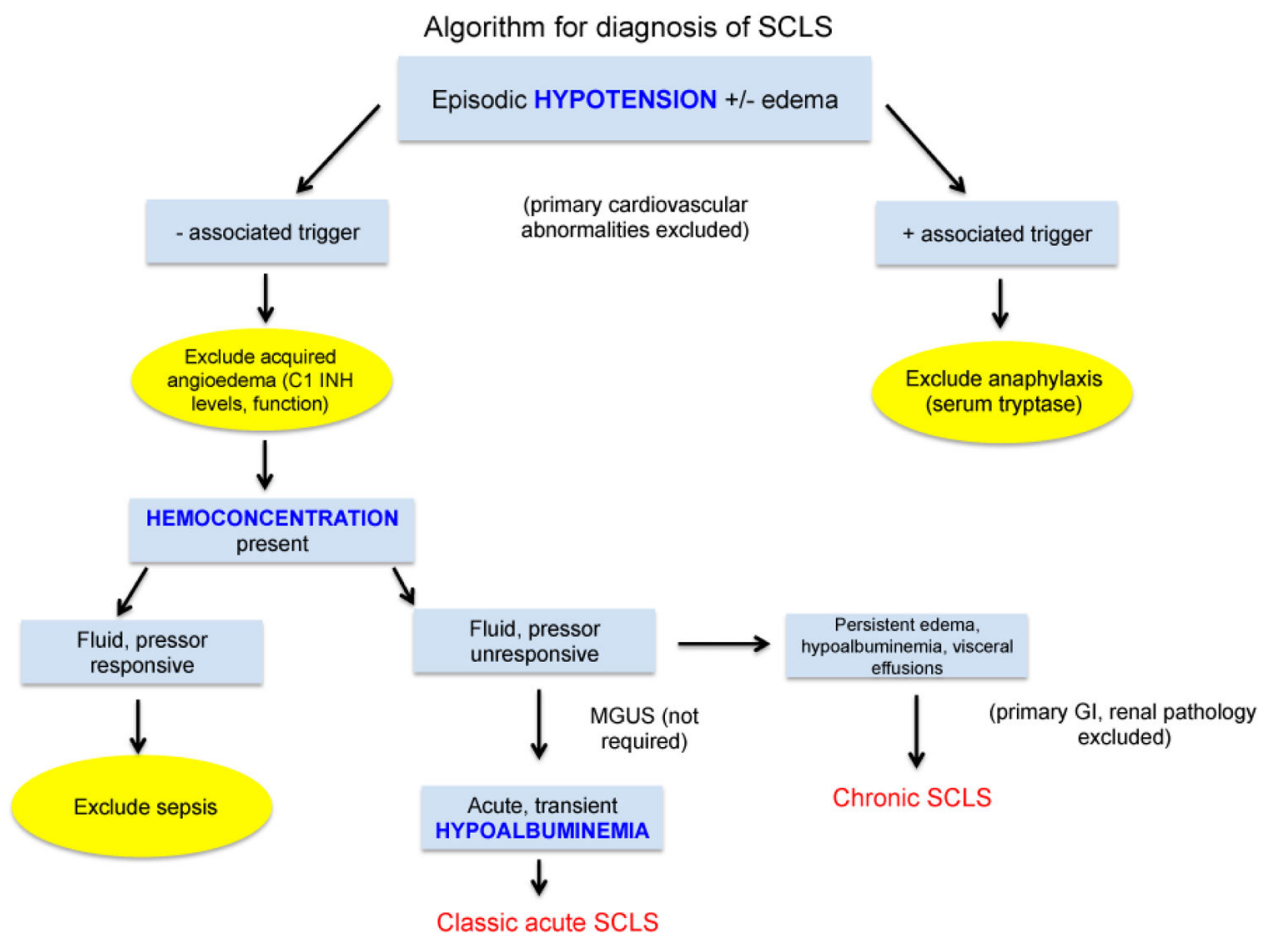

Figure 1.

Clinical considerations in the prospective diagnosis of SCLS. After exclusion of primary cardiovascular and/or allergic causes, a diagnosis of SCLS should be entertained in patients with unexplained, transient hypotension and/or peripheral edema. Systemic anaphylaxis and hereditary and/or acquired angioedema can be excluded by measurement of serum tryptase during the acute episode and quantitative and functional assays for the complement component 1 esterase inhibitor ( $\mathrm{C} 1 \mathrm{INH})$. Although presumptive treatment for sepsis is prudent in the undiagnosed SCLS patient during the first severe episode, the hypotension and hemoconcentration of SCLS are typically refractory to intravenous fluid resuscitation, which exacerbates peripheral edema. Hypoalbuminemia due to protein extravasation is a hallmark of classic acute SCLS whereas low serum albumin levels and edema that does not resolve between episodes should prompt the diagnosis of "chronic" SCLS. MGUS is not universally present in SCLS and is therefore not required for the diagnosis. 
A

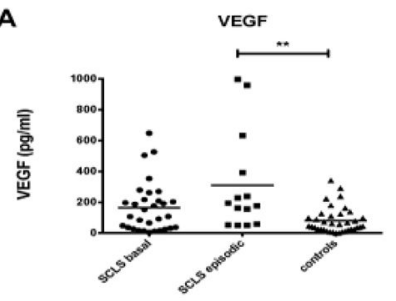

C

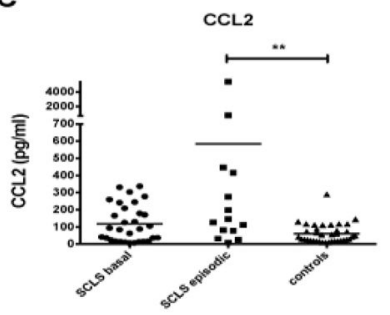

E

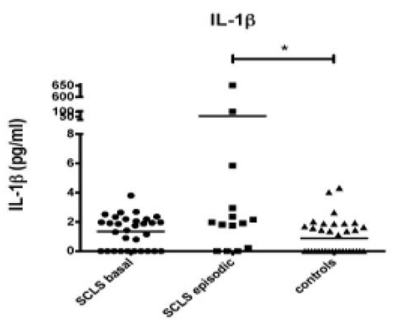

G

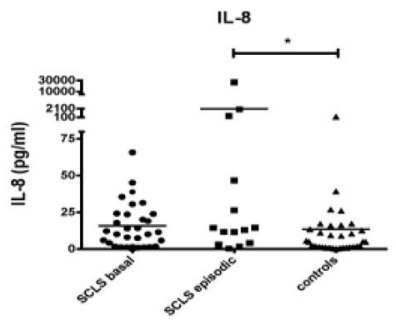

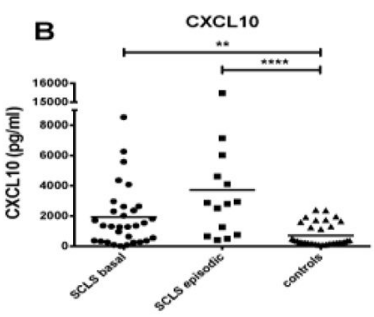

D

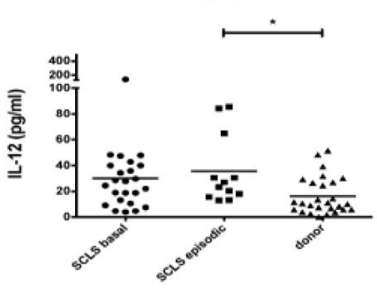

$\mathbf{F}$

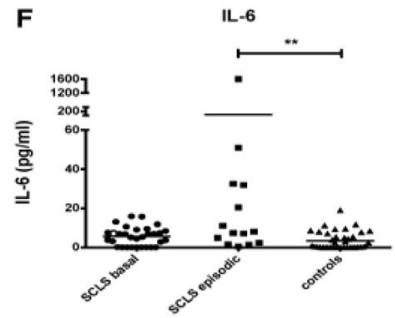

H

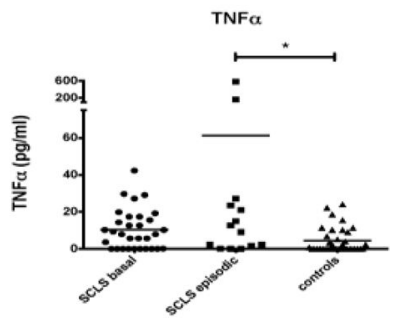

Figure 2.

Cytokine profiles of SCLS sera. (A-H) Serum cytokines were measured using a Bio-Plex Pro human multiple cytokine assay kit: VEGF (A), CXCL10 (B), CCL2 (C), IL-12 (D), IL-1 $\beta(\mathrm{E}), \mathrm{IL}-6(\mathrm{~F}), \mathrm{IL}-8(\mathrm{G})$ and TNFa $(\mathrm{H})(* P<0.05, * * P<0.01, * * * * P<0.00001$ Kruskal-Wallis). 
A
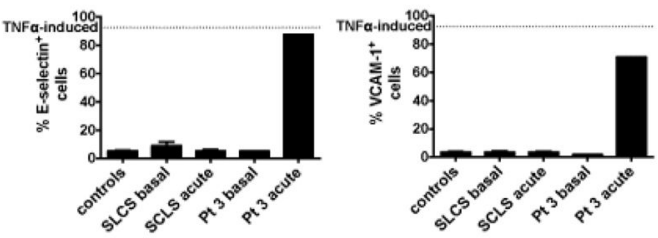

B
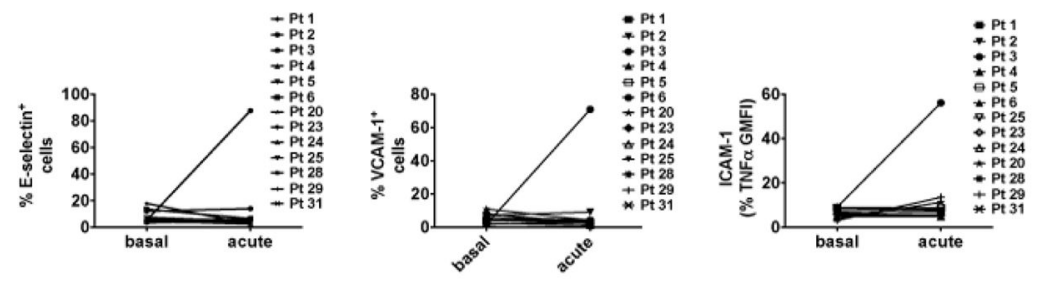

C

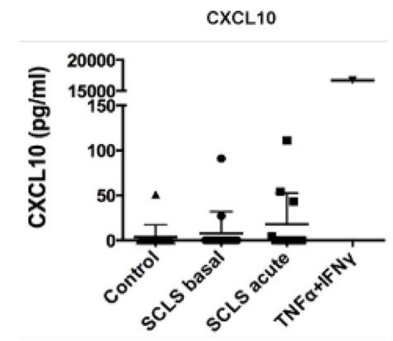

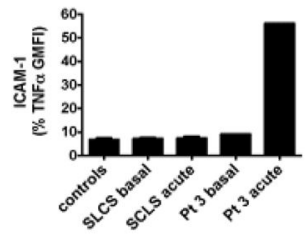$$
\text { . }
$$ 
A
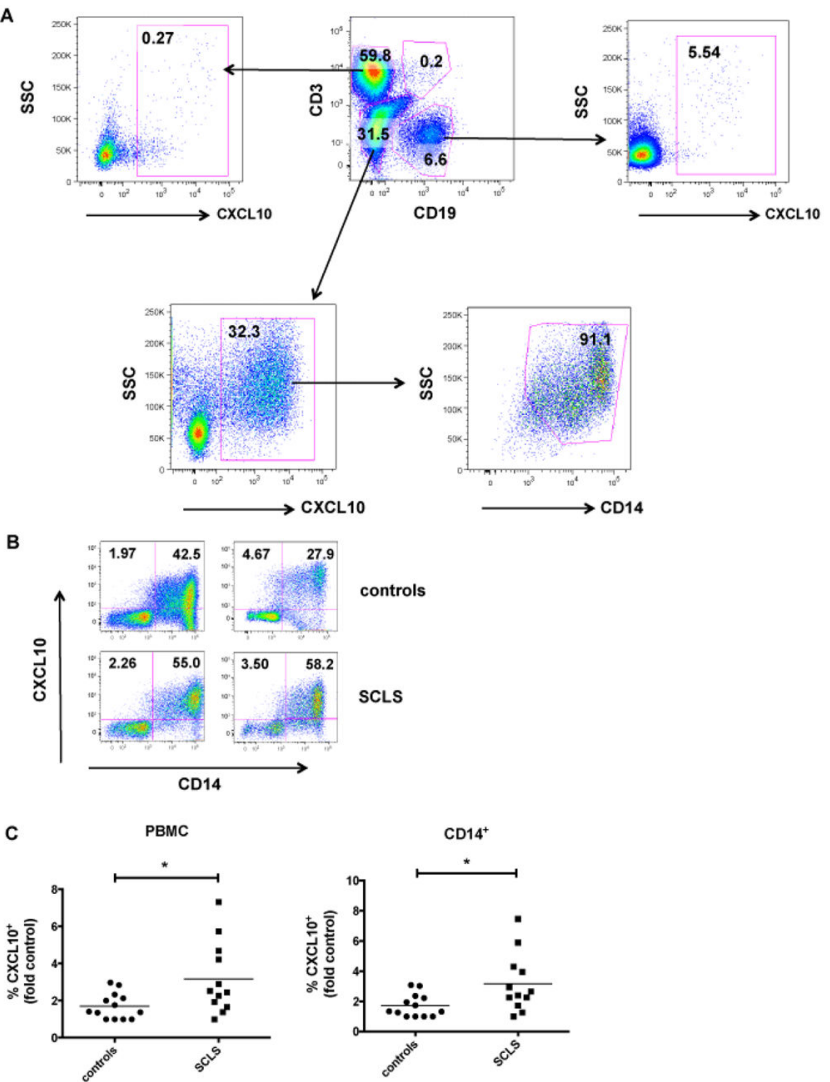

Figure 4.

Abnormal IFN $\gamma$-induced CXCL10 production by PBMCs in SCLS. PBMCs were incubated with IFN $\gamma$ and analyzed for CXCL10 production by flow cytometry. (A) Percentages of CXCL10+ cells among T lymphocytes (CD3+), B lymphocytes (CD19+), and monocyte (CD14+) populations from a representative healthy subject. (B-C) Representative flow plots (B) and analysis of CXCL10+ PBMCs and monocytes from SCLS subjects $(\mathrm{n}=12)$ and healthy subjects $(\mathrm{n}=14)$, presented as the fold control of the lowest value from a healthy donor for each individual experiment, set as ' 1 '. ${ }^{*} P=0.02$, unpaired $t$ test. 


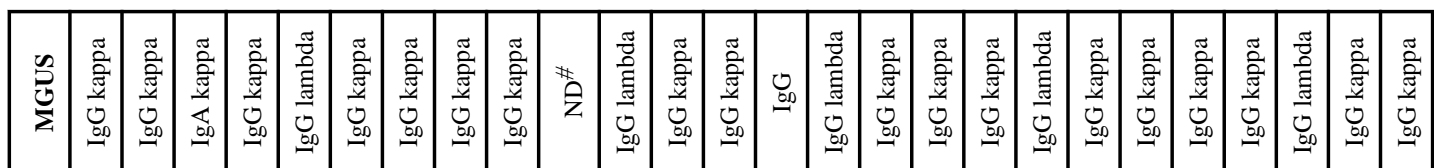

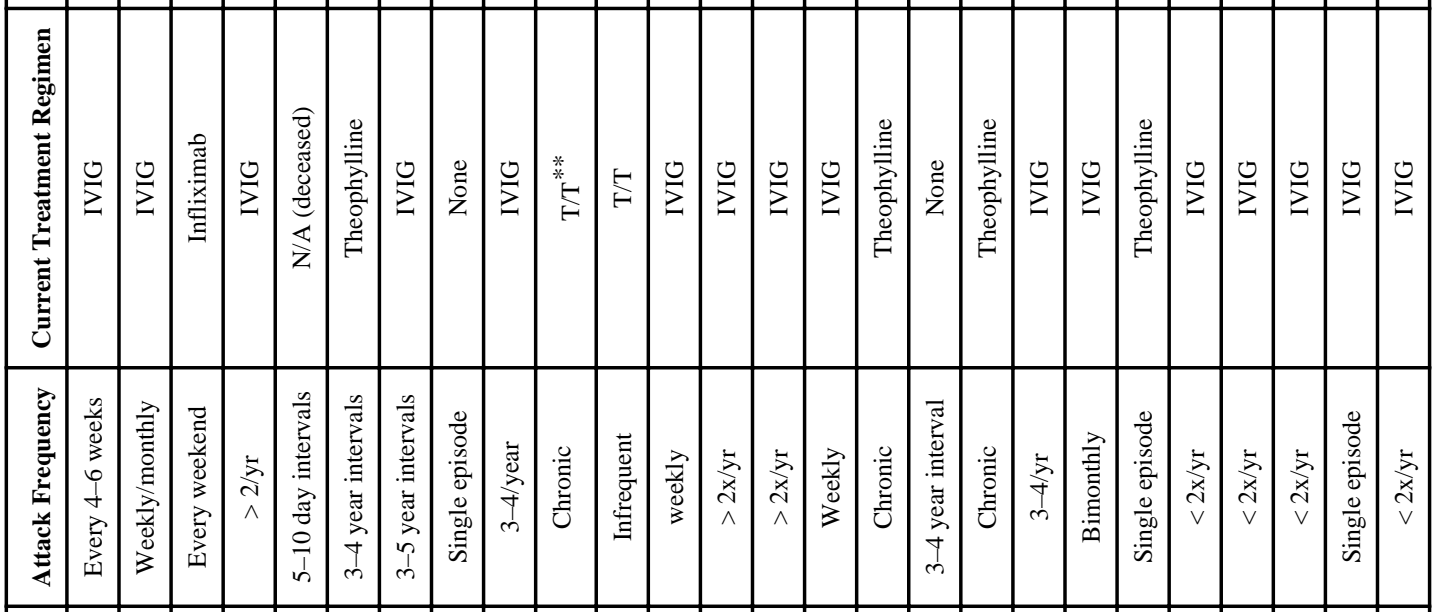

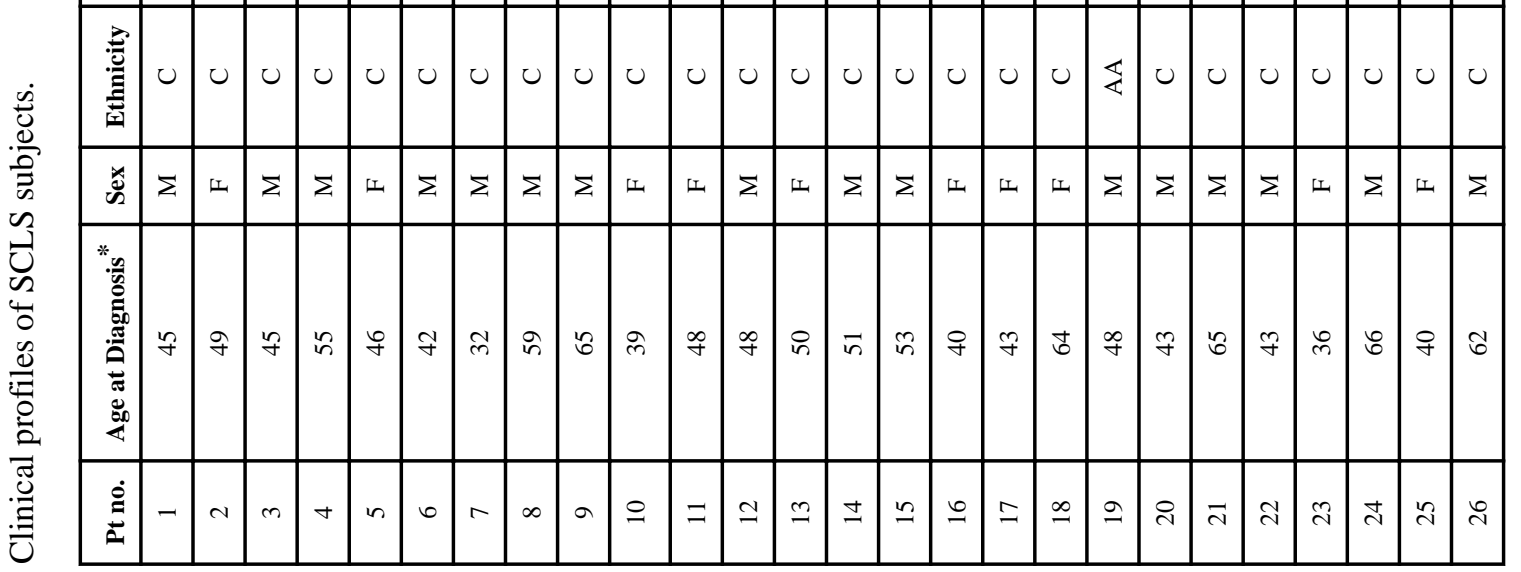




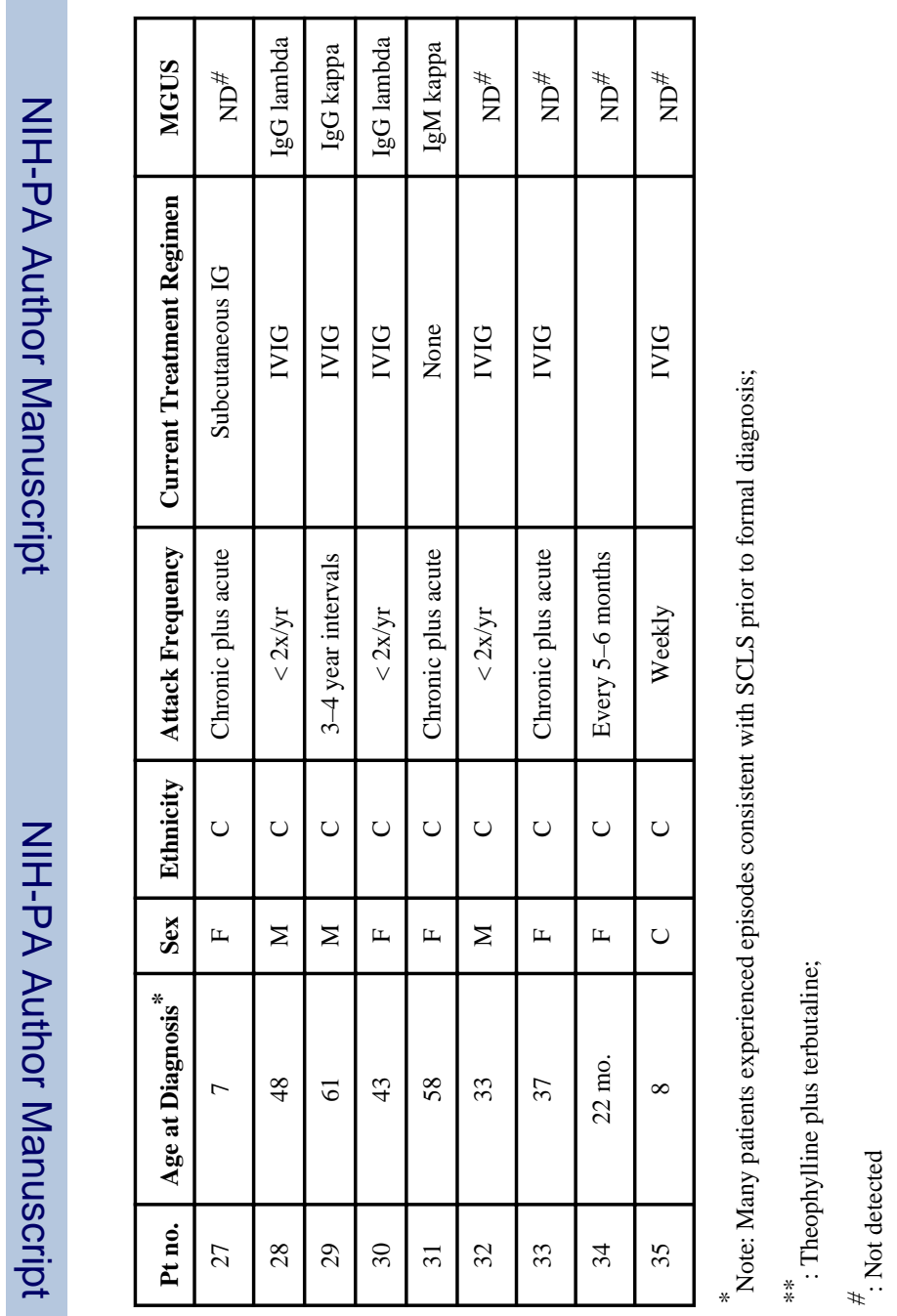

J Clin Cell Immunol. Author manuscript; available in PMC 2014 November 15. 